CONSEJO DE ESTADO 



\title{
EL DICTAMEN DEL CONSEJO DE ESTADO SOBRE EL «TRATADO POR EL QUE SE ESTABLECE UNA CONSTITUCIÓN PARA EUROPA»
}

\author{
MARÍA SALVADOR MARTÍNEZ \\ Prof. Ayudante de Derecho Constitucional (UNED) \\ SUMARIO \\ I. Los antecedentes. \\ II. El cuerpo del dictamen.
}

En sesión celebrada el 21 de octubre de 2004, la Comisión Permanente del Consejo de Estado emitía dictamen sobre el «Tratado por el que se establece una Constitución para Europa», ${ }^{1}$ un dictamen de enorme relevancia, no sólo por su objeto — un tratado internacional que supone un paso determinante en el proceso de integración europea-, sino también por las consideraciones que en él se hicieron y el debate que generaron. ${ }^{2}$ Antes de emitir este dictamen, el Consejo de Estado ya se había pronunciado en su Memoria del año 2003 sobre el entonces proyecto de Tratado, de modo que las principales reflexiones que en aquel momento se hicieron han sido posteriormente recogidas en el dictamen que nos ocupa. Así pues, en el análisis y exposición del mismo, haremos referencia, allí donde sea necesario, a la citada Memoria.

De acuerdo con la estructura característica de los dictámenes del Consejo de Estado, el texto del dictamen sobre el «Tratado por el que se establece una Constitución para Europa» consta de dos partes bien diferenciadas: una primera, en la que se describen los antecedentes del expediente, y la segunda, que contiene el dictamen propiamente dicho.

1 El texto que analiza el Consejo de Estado es el acordado el 18 de junio de 2004 por los jefes de Estado y de Gobierno de los Estados miembros de la Unión Europea. El texto definitivo, incorporadas las correcciones de estilo de los traductores, se ha publicado en el Diario Oficial, C 310, con fecha de 16 de diciembre de 2004.

2 Dictamen $n^{\circ}$ 2544/2004 del Consejo de Estado, relativo al «Tratado por el que se establece una Constitución para Europa». 
Constitución y, a la luz de este artículo, realiza las reflexiones sobre el contenido del Tratado que considera necesarias (págs. 31 a 42); en el tercero, se pronuncia sobre la necesidad de verificar la constitucionalidad del Tratado y señala el que, a su juicio, es el principal problema de compatibilidad que existe entre el Tratado y nuestra Constitución (págs. 43 a 48); y, en el cuarto y último apartado, aconseja consultar al Tribunal Constitucional, en aplicación del art. 95.2 de la Norma Suprema, y presenta algunas propuestas sobre una posible reforma constitucional (págs. 49 a 51).

\section{La NATURALEZA DEL «TRATAdo POR EL QUE SE ESTABLECE UNA CONSTITUCIÓN PARA EuROPA»}

Analizado el instrumento sometido a consulta, el Consejo de Estado afirma que se trata de un Tratado Internacional, y, más concretamente, de un «Tratado de integración supranacional». Según se advierte, el propio preámbulo del texto indica que «es continuación de la obra realizada por los Tratados comunitarios» y que «pretende profundizar en las bases de la integración». En palabras del Consejo de Estado, «el Tratado viene a dar un nuevo fundamento a la Unión por cuanto sustituye a los tratados constitutivos de la Comunidad Europea y de la Unión Europea, que deroga (art. IV-437), y crea una Unión Europea a la que los Estados miembros atribuyen competencias para alcanzar sus objetivos comunes (art. I-1), con personalidad jurídica única (art. I-7), y que sucede a la Unión Europea constituida por el Tratado de Maastricht y a la Comunidad Europea (art. IV-438)».

En relación con la categoría de «tratado de naturaleza supranacional», el Consejo se remite a lo expresado en la Memoria del Consejo de Estado de 2003, en la que, en relación con el entonces proyecto de Tratado, se hacían las siguientes observaciones.

El Consejo de Estado recordaba en dicha Memoria cómo en repetidas ocasiones había afirmado que «el Derecho comunitario no es ni nacional ni internacional, sino un orden distinto y autónomo», ${ }^{3}$ pero que «cada vez se aleja mas de lo típicamente internacional para adquirir rasgos propios del derecho constitucional estatal, hasta el punto de ser, como ha dicho el Tribunal Constitucional, a ciertos efectos, derecho interno» ${ }^{4}$. Entiende el Consejo que la elaboración de este proyecto de tratado constituye un paso más, un paso determinante, en ese proceso de alejamiento de lo típicamente internacional y de «constitucionalización de la Unión Europea», y así lo afirmaba en la Memoria: «la Unión Europea está en trance de novar profundamente sus estructuras intensificando la naturaleza constitucional de sus instituciones, naturaleza hace ya tiempo afirmada por el Tribunal de Justicia de las Comunidades Europeas al considerar a los tratados fundacionales como una Carta Constitucional básica». ${ }^{5}$

Afirmado lo anterior, el Consejo de Estado no dudaba sin embargo en calificar el Tratado en proyecto como «tratado de integración supranacional», ya que es «en cuanto a la forma, un texto escrito único, simplificador de los diferentes tratados anteriores, racionalizador de las doctrinas acuñadas por la jurisprudencia del Tribunal de Justicia de las Comunidades Europeas, con pretensiones de supremacía jurisdiccionalmente garantizada y de estabilidad asegurada con una cierta rigidez en cuanto a su reforma se refiere; y, por lo que hace al fondo, un texto destinado a definir los valores de la Unión, a regular sus instituciones, a precisar las competencias y, probablemente, a garantizar, con mayor o 


\section{LOS ANTECEDENTES}

El Consejo de Estado ha ordenado los elementos que integran los antecedentes en tres apartados, cada uno de los cuales tiene un contenido diferente.

1. En el primer apartado el Consejo de Estado hace un breve repaso del itinerario seguido por el proyecto de «Tratado por el que se establece una Constitución para Europa», desde la Declaración de la Conferencia intergubernamental de Niza del año 2000, hasta su aprobación en junio de 2004. A continuación se describen la estructura y el contenido del Tratado, lo cual, dada la extensión del mismo, hace que éste sea el apartado más largo de los que integran los antecedentes (págs. 2 a 27).

2. En el segundo apartado se relacionan los informes que figuran en el expediente, todos ellos favorables, señalándose los aspectos del Tratado que se han destacado en cada uno de los informes (págs. 27 a 29).

3. En el tercer apartado se da cuenta del informe-propuesta que sobre el Tratado emitió el Gabinete de Tratados de la Secretaría General Técnica del Ministerio de Asuntos Exteriores y de Cooperación. Dicho informe señalaba, respecto al proceso de ratificación, que estaba previsto que el día 29 de octubre, en Roma, el Presidente del Gobierno firmase el Tratado, tras lo cual se iniciaría el proceso de ratificación por las Cortes que, «de acuerdo con la práctica establecida y con lo dispuesto en el art. 93 de la Constitución», sería a través de una ley orgánica, añadiendo que el Presidente del Gobierno había anunciado su intención de convocar previamente un referéndum. Finalmente, señalaba el informe que el Gobierno y las Cámaras pueden consultar al Tribunal Constitucional, al amparo del art. 95.2 de la Constitución, aunque «a juicio del equipo técnico responsable por parte española de las negociaciones para la conclusión del Tratado, no existe ninguna contradicción entre el texto del Tratado acordado y la Constitución Española. El artículo I-6 (primacía del derecho comunitario) se limita a reflejar la actual jurisprudencia del Tribunal de Justicia y el art. I-5 proclama la obligación por parte de la Unión de respetar las estructuras fundamentales políticas y constitucionales de sus Estados miembros» (págs. 29 y 30).

\section{EL CUERPO DEL DICTAMEN}

Por lo que se refiere al dictamen propiamente dicho, el cuerpo del mismo se estructura en cuatro apartados. En el primero, el Consejo afirma la naturaleza de «tratado de integración supranacional» del texto sobre el que debe pronunciarse (págs. 30 y 31); en el segundo, reconoce que la vía de ratificación del Tratado es la indicada por el art. 93 de la

3 Dictamen $n^{\circ} 47.939$ de 13 de junio de 1985; doctrina legal (v.gr. Dictamen $n^{\circ}$ 421/92); Memorias del Consejo de Estado de 1985 y 1992.

4 STC $165 / 1994$

5 El Consejo cita aquí el Dictamen no 1/1992 sobre el EEE. 
que atribuyan competencias a organizaciones supranacionales, pero, también, que de dicho precepto derivan ciertos límites a la posibilidad de autorizar un tratado de ese tipo. En primer lugar, «la atribución lo es del ejercicio de competencias, no de su titularidad» ${ }^{10}$, algo que, en este caso, se pone claramente de manifiesto en el carácter revocable de la cesión a través del procedimiento previsto en el Tratado para la retirada voluntaria de la Unión Europea (art. I-60). En segundo lugar, la atribución debe estar referida a competencias derivadas de la Constitución, es decir, competencias normativas, ejecutivas y judiciales, que permitan a la organización supranacional adoptar decisiones - no necesariamente por unanimidad - aplicables a los Estados miembros y a los particulares, como ocurre en el caso del Tratado sometido a examen. ${ }^{11} \mathrm{Y}$, en tercer lugar, la atribución lo es «de competencias», y no de «las» competencias, de lo que resulta que no cabe, ni una atribución en bloque que suponga un vaciamiento de las competencias estatales o la quiebra de la pervivencia de la organización política del Estado, ni tampoco una atribución indeterminada de competencias; pues bien, es en relación con este tercer límite donde se detiene el Consejo de Estado.

De un lado, a la vista de que el propio Tratado declara el respeto por la Unión Europea de la «identidad nacional» de los Estados miembros, «inherente» a sus estructuras fundamentales políticas y constitucionales, así como el respeto a las «funciones esenciales del Estado» (art. I-5), entiende el Consejo de Estado que en este caso no se realiza atribución en bloque alguna que suponga un vaciamiento de las competencias estatales o la quiebra de la pervivencia del Estado.

De otro, en cuanto al grado de determinación de las competencias que se atribuyen, reconoce el Consejo que, hasta ahora, cabían ciertas dudas sobre si las mismas estaban suficientemente definidas. El sistema competencial de la Unión se ha caracterizado por la progresividad, el casuismo, la complejidad y por tener contornos difusos o poco definidos; las competencias se atribuían conforme a un «método finalista» y su alcance se determinaba en función de los objetivos a cumplir; se estableció una cláusula de flexibilidad (art. 308 TCE); el Tribunal de Justicia, por su parte, apoyó la expansión de las funciones de la Unión

6 Recuerda el Consejo de Estado lo afirmado en memorias e informes anteriores: que de la lectura del texto constitucional (del art. 93) se deduce la existencia de dos tipos de tratados internacionales, los de cooperación internacional y los de integración supranacional, y que estos últimos se caracterizan por «la cesión sistemática del ejercicio de competencias a un nuevo sujeto, que las desempeña de manera autónoma mediante la producción extraestatal de normas con efecto directo sobre los ciudadanos de los Estados miembros». Entiende el Consejo que una mayor integración supranacional, incluso de carácter «constitucional», como es la que se busca con este proyecto, debe tener lugar por la vía que nuestra Constitución prevé para ello, la vía convencional del art. 93. De ello deriva una importante consecuencia que se explicita en el proyecto y que el Consejo quiere subrayar: pese a la interpretación que en su momento se hizo de los Tratados fundacionales, la integración supranacional es reversible, «puesto que su título legitimador siguen siendo las prescripciones constitucionales internas y la soberanía estatal que expresan».

7 Recordemos que el art. 93 CE establece: «Mediante ley orgánica se podrá autorizar la celebración de tratados por los que se atribuya a una organización o institución internacional el ejercicio de competencias derivadas de la Constitución».

8 Se citan los Dictamen $n^{\circ}$ 5.072/97, relativo al Tratado de Ámsterdam; no 880/2001, relativo al Tratado de Niza; $\mathrm{y}^{\circ} 1.173 / 2003$, relativo al acta de adhesión de nuevos miembros.

9 Citando el Dictamen n ${ }^{\circ}$ 5.072/97 del Consejo de Estado. 
menor grado de eficacia, un catálogo de derechos a los ciudadanos europeos tal como esta categoría fue formulada en el Tratado de Niza». Por lo tanto, cualquiera que sea la denominación del proyecto (Tratado por el que se establece una Constitución), y a pesar de que esté destinado a establecer un marco institucional de la Unión Europea con pretensiones constitucionales, afirmaba el Consejo entonces, el texto del proyecto ha de calificarse como «tratado de integración supranacional». ${ }^{6}$

\section{Reflexiones a la luZ DEL ARTíCUlo 93 de la CONSTITUCióN}

Si el Tratado sometido a examen es un «tratado de integración supranacional», resulta entonces claro que su ratificación debe realizarse a través de la vía establecida en el art. 93 de la Constitución. ${ }^{7}$ Recuerda el Consejo que, como ya se ha indicado en diversos dictámenes anteriores, ${ }^{8}$ la orientación internacionalista de la Constitución, que se manifiesta en varios lugares de la misma, alcanza su máxima intensidad en el art. 93, que el constituyente redactó teniendo en mente la eventual adhesión de España a las Comunidades Europeas, y que, por ello, ésta ha sido la vía utilizada para la celebración de los sucesivos Tratados comunitarios, «la vía específica, cualificada e idónea para que España vaya cubriendo las diversas etapas de la construcción europea, de cuya naturaleza evolutiva era sin duda consciente el legislador constitucional»?

Desde este punto de vista, el Consejo de Estado considera necesario analizar el significado de este nuevo Tratado en el proceso de construcción europea, señalando las innovaciones fundamentales que trae consigo: reconocimiento de personalidad jurídica a la Unión Europea, simplificación y redefinición de las fuentes del derecho comunitario, su extensión a gran número de ámbitos, potenciación del Parlamento Europeo, reconocimiento de un cierto derecho de iniciativa legal a los ciudadanos, atribución de determinadas funciones a los parlamentos nacionales, nueva definición de la mayoría cualificada en el Consejo Europeo y en el Consejo de Ministros, etc.

De entre todos los cambios que introduce el Tratado sometido a consulta, sólo son dos los que el Consejo de Estado considera necesario examinar detalladamente a la luz del art. 93 de la Constitución, a fin de verificar si la vía señalada por dicho artículo es suficiente e idónea: el primero, la atribución de competencias a la Unión Europea, sobre la que hasta el momento se han suscitado ciertas dudas; y, el segundo, la llamada Carta de Derechos Fundamentales, por su trascendencia en el proceso de integración.

\subsection{LA ATRIBUCIÓN DE COMPETENCIAS A LA UNIÓN EUROPEA}

Por lo que se refiere a la atribución de competencias a la Unión Europea, recuerda el Consejo de Estado que el art. 93 de la Constitución permite la celebración de tratados

10 Cita aquí el Consejo de Estado la Declaración de 1 de julio de 1992 del Tribunal Constitucional, FJ 4.

11 Recuerda el Consejo de Estado sus Dictámenes 1.376/1994 y 1.173/2003, en los que así lo reconoce, y cita también la Declaración de 1 de julio de 1992 del Tribunal Constitucional. 
iguales a los que les confiere el Convenio Europeo de Derechos Humanos, así como que los derechos que resultan de las tradiciones constitucionales comunes serán interpretados con acuerdo con dichas tradiciones (art. II-112.3 y 4). Añade el Consejo que la Carta invoca el Convenio Europeo de Derechos Humanos, al que también nos remite nuestra propia Constitución, a través del art. 10.2, lo que confirma la identidad sustancial de la Carta con el orden de valores, derechos y libertades proclamados por nuestra Constitución.

En segundo lugar, la regulación que hace la Carta de los límites de los derechos (art. II-112.1) es equiparable a la que contiene nuestra Constitución: cualquier limitación debe ser establecida por ley, respectar el contenido esencial y superar el conocido «test de proporcionalidad». Y, además, la Carta introduce una prohibición del abuso de derecho (art. II-114) similar a la de otras declaraciones internacionales de derechos.

Incorporada la Carta al Tratado, advierte el Consejo de Estado que los órganos jurisdiccionales internos se encontrarán vinculados por la misma en la aplicación del derecho comunitario, de modo tal que se introducirá así un cierto control difuso de las normas nacionales —incluso con rango de ley_ que resulten incompatibles con la Carta, y que la consecuencia de la incompatibilidad, cuando se dé, será la inaplicación de la norma nacional. A juicio del Consejo, este tipo de control puede incidir en la labor que corresponde al Tribunal Constitucional, aunque éste mantenga el monopolio de la declaración de nulidad de las leyes. ${ }^{12}$

En relación con lo anterior, y a pesar de haber concluido que las disposiciones de la Carta no van a producir colisiones o discordancias con la configuración que la Constitución Española hace de los derechos y libertades, reconoce el Consejo que la coexistencia de tres regímenes o parámetros en la tutela de los derechos fundamentales (Constitución, Convenio europeo y Carta) determinará en el futuro, si el Tratado entra en vigor, un proceso de influencias mutuas no exento de problemas jurídicos, que, a juicio del Consejo de Estado, corresponde aclarar al Tribunal Constitucional en lo que se refiere al sentido de la vinculación de las autoridades españolas por la Carta, las relaciones de ésta con nuestro sistema constitucional de derechos y libertades, y el modo de depuración de las normas que la contradigan. Debemos señalar aquí que esta última reflexión del Consejo de Estado, relativa a la tarea del Tribunal Constitucional, sirvió de base para la consulta sobre la constitucionalidad de los arts. II-111 y 112 del Tratado que plantearía posteriormente el Gobierno al citado Tribunal.

Por último, el Consejo cree preciso hacer una referencia concreta a la ciudadanía de la Unión, integrada por los cuatro derechos procedentes del Tratado de la Unión Europea (derecho a libre circulación y residencia, derecho de sufragio activo y pasivo, derecho a protección diplomática y consular, y derecho de petición) y otros dos derechos que el nuevo Tratado añade: el derecho a una buena administración y el derecho de acceso a los documentos de las instituciones comunitarias. Respecto a esta nueva configuración de la ciudadanía europea, afirma el Consejo que «los nuevos derechos están referidos al ámbito de actuación de los poderes de la Unión y se sitúan en plena consonancia con los derechos de los administrados según las tradiciones constitucionales de los Estados miembros», y recuerda la Declaración de España relativa a la definición del término «nacionales», así como que, conforme al Tratado, la ciudadanía de la Unión se añade a la ciudadanía nacional sin sustituirla ni interferirla, de todo lo cual concluye que no parecen existir "problemas de acoplamiento» entre las nuevas disposiciones sobre la ciudadanía europea y el ordenamien- 
a través de principios como el de efectividad, y de doctrinas como la del paralelismo entre las competencias internas y externas de la Comunidad, y la del reconocimiento del carácter dinámico del ordenamiento comunitario; mientras que, por otro lado, se intentaba limitar el ejercicio de competencias de la Unión mediante los principios de subsidiariedad y proporcionalidad.

Frente a este panorama, reconoce el Consejo de Estado que el «Tratado por el que se establece una Constitución para Europa» revisa profundamente la formulación de las competencias de la Unión, para clarificar y precisar el marco competencial de la misma, reduciendo el amplio margen de interpretación que los tratados habían permitido hasta ahora. El nuevo Tratado expresa los principios rectores del reparto de poderes (arts. I-1 y I-13); clasifica las competencias, simplifica los instrumentos jurídicos de actuación, y determina los tipos de actos que deben utilizarse en cada caso y los procedimientos que han de aplicarse; distingue tres categorías de competencias, las exclusivas (art. I-13), las compartidas (art. I-14) y las de realización de acciones de apoyo, coordinación o complemento (art. I-17); incorpora «normas competenciales negativas» (como la contenida en el art. II-111); y, finalmente, recoge tanto la cláusula de flexibilidad del art. 308 TCE, que exige ahora la previa aprobación del Parlamento Europeo (art. I-18), como los principios de proporcionalidad y subsidiariedad, atribuyendo un mayor control a los parlamentos nacionales y al Tribunal de Justicia (Protocolo sobre la aplicación de los principios de subsidiariedad y proporcionalidad, anexo al Tratado).

Así pues, concluye el Consejo de Estado que, si anteriormente el sistema de atribución de competencias no fue óbice para la ratificación de los correspondientes tratados, con menor motivo habría de serlo ahora.

\subsection{La Carta de Derechos Fundamentales}

En relación con la Parte II del Tratado, que incorpora la Carta de Derechos Fundamentales de la Unión Europea y culmina el proceso de sometimiento de las instituciones comunitarias a un catálogo de derechos común a las tradiciones constitucionales de los Estados miembros, considera el Consejo de Estado que, dejando a un lado las dificultades que la práctica evidencie en el futuro, parece que las disposiciones de la Carta no van a producir colisiones o discordancias con la configuración que nuestra Constitución hace de los derechos y libertades. A esta conclusión llega tras constatar los siguientes datos.

En primer lugar, el Tratado dispone que la Carta vincula únicamente a los Estados miembros cuando apliquen el derecho comunitario y que no implicará ampliación alguna de las competencias atribuidas a la Unión (art. II-111); establece que las disposiciones de la Carta no pueden interpretarse como limitativas o lesivas de los derechos reconocidos por las Constituciones nacionales y los convenios internacionales firmados por los Estados miembros (art. II-113); y precisa que el sentido y alcance de los derechos de la Carta serán

12 El Dictamen recuerda que el Tribunal de Justicia ha afirmado que las jurisdicciones nacionales están obligadas a inaplicar cualquier norma nacional que resulte incompatible con el derecho comunitario (STJCE de 9 de marzo de 1978), y que el Tribunal Constitucional ha confirmado que si una ley es contraria al derecho comunitario sólo puede ser inaplicada mediante el planteamiento de una cuestión prejudicial (STC 58/2004). 
a) En primer lugar, en relación con las «mutaciones constitucionales» que se han ido generando desde la incorporación de España a las Comunidades, afirmaba el Consejo de Estado que sería bueno abordarlas con tiempo suficiente, tanto para conseguir la modificación puntual del proyecto si fuese conveniente y posible, como para preparar las modificaciones legales e incluso constitucionales oportunas, porque, aunque la mutación constitucional esté reconocida como vía de adecuación de la Constitución a la realidad, el significado que se atribuya a aquélla no puede llegar a ser incompatible con la norma escrita. Se refería entonces el Consejo a las principales «mutaciones constitucionales» consecuencia de la integración europea, relativas la mayoría a la llamada constitución económica, ${ }^{15}$ por ejemplo, al significado de «la modernización y desarrollo de todos los sectores económicos y, en particular, de la agricultura, de la ganadería y de la pesca» (establecida en el art. $130 \mathrm{CE}){ }^{16}$ y al de la intervención pública en la economía (que prevén el art. $128 \mathrm{CE}$ y otros). ${ }^{17}$

b) En segundo lugar, se hacía referencia en dicha Memoria a las cuestiones que podrían plantear mayores problemas de constitucionalidad: el principio de primacía, al que luego nos referiremos, la aplicación por el juez nacional del derecho comunitario, y a la incidencia del nuevo Tratado en la distribución de competencias entre el Estado central y las Comunidades Autónomas.

- En cuanto a la aplicación por las jurisdicciones estatales del Derecho comunitario, recuerda el Consejo que actualmente «el juez ordinario es juez del Derecho comunitario, encargado de garantizar la primacía de éste excluyendo, en su caso, la aplicación de la norma nacional y con la correlativa obligación de plantear la cuestión prejudicial ante el Tribunal de Justicia. Por el contrario, el Tribunal Constitucional no es juez del Derecho comunitario y no lo aplica ni cuida de su aplicación, de manera que tampoco tiene en cuenta su toma en consideración por el juez ordinario a los efectos de conceder el amparo». Aunque el Tribunal Constitucional no ha podido dejar de tener en cuenta las normas comunitarias al interpretar ciertos aspectos de la Norma fundamental, cree el Consejo que «la superación de esta situación un tanto disfuncional podría encontrarse haciendo del derecho primario de la Unión —en el futuro posiblemente el tratado constitucional- canon de constitucionalidad, integrándolo en el correspondiente bloque mediante la reforma del artículo 28 de la Ley Orgánica del Tribunal Constitucional (LOTC)». ${ }^{18}$

- En cuanto a la incidencia del proyecto de Tratado en la distribución de competencias prevista por la Constitución española, de acuerdo con la doctrina legal acuñada por el Consejo de Estado y las reiteradas consideraciones contenidas en las Memorias desde el año 1985, entiende el Consejo que el princi-

13 El citado art. $95 \mathrm{CE}$ establece que: «La celebración de un tratado internacional que contenga estipulaciones contrarias a la Constitución exigirá la previa revisión constitucional. El Gobierno o cualquiera de las Cámaras puede requerir al Tribunal Constitucional para que declare si existe o no esa contradicción». 
to español.

\section{LA NECESIDAD DE VERIFICAR LA CONSTITUCIONALIDAD DEL TRATADO}

Llegado a este punto, una vez que ha determinado que la vía para ratificar el nuevo Tratado es la señalada por el art. 93 de la Constitución, el Consejo considera que «ha de analizarse si el art. 95 de la Constitución pudiera vedar — bien que de modo inicial y condicional pero inmediato- la prestación del consentimiento del Estado incluso por la vía del art. $93 »^{13}$, es decir, que es preciso contrastar el Tratado con la Constitución Española, a fin de comprobar si existiera algún tipo de contradicción entre ambos. En ese sentido, recuerda lo afirmado por el Tribunal Constitucional sobre el significado del citado art. 95, que «el tenor y el sentido mismo del art. 95, aplicable a todo tipo de tratados, excluyen con claridad el que mediante cualquiera de ellos puedan llegar a ser contradichas o excepcionadas las reglas constitucionales ... (...) ... (Las Cortes) pueden ceder o atribuir el ejercicio de competencias derivadas de la Constitución, no disponer de la Constitución misma, contrariando o permitiendo contrariar sus determinaciones, pues ni el poder de revisión constitucional es una competencia cuyo ejercicio fuera susceptible de cesión, ni la propia Constitución admite ser reformada por otro cauce que no sea el de su Título X, esto es, a través de los procedimientos y con las garantías allí establecidas, y mediante la modificación expresa de su propio texto». ${ }^{14}$

Se trata de una preocupación que ha expresado ya el Consejo de Estado en otras ocasiones. En la Memoria de 2003, al hacer referencia al entonces proyecto de Tratado, el Consejo recordaba la experiencia de la ratificación del Tratado de Maastricht y señalaba la conveniencia de analizar con antelación suficiente la compatibilidad del proyecto con la Constitución. Ya entonces señalaba el Consejo que, a la hora de celebrar un tratado más integrador que el de Maastricht, como es el «Tratado por el que se establece una Constitución para Europa», debían evitarse tanto la improvisación de soluciones como la excesiva simplificación de los problemas en presencia. En este sentido, apuntaba el Consejo que no se trata de realizar «un mero cotejo del nuevo Tratado con la letra de nuestra Constitución para detectar eventuales contradicciones puntuales entre uno y otro texto. Antes bien, según ya se hizo en importantes Estados miembros de la Unión, tal vez se requiera plantear con mayor amplitud la compatibilidad del nuevo tratado de integración con la propia Constitución estatal, para determinar si la voluntad política del Estado en ser Parte en dicho tratado requiere o no la previa reforma de la Constitución en cumplimiento de su artículo 95».

En relación con ese «control de constitucionalidad» previo del Tratado, el Consejo de Estado se refería en su Memoria de 2003 a dos tipos diferentes de problemas.

14 Declaración del Tribunal Constitucional, de 1 de julio de 1992, relativa al Tratado de Maastricht.

15 Aunque el Consejo también se refería al desapoderamiento parcial de las Cortes Generales (el Consejo ha considerado «actos debidos» el ejercicio de las competencias parlamentarias cuando venían determinadas por el Derecho comunitario, cf. Dictamen no 51.692 de 1988) y a la reinterpretación de importantes competencias estatales, ya por su transferencia a instancias de la Unión (como es el caso de la política monetaria del artículo 149.1. $11^{\mathrm{a}}$ ), ya por el cambio en el significado de los términos en que venían enunciadas (como es el caso de las referentes a sanidad y comercio exterior, arts. 149.1, $10^{\mathrm{a}}$ y $16^{\mathrm{a}}$ ), ya, en fin, por su necesaria reinterpretación para mantener el propio sistema competencial previsto en la Constitución (como el monopolio estatal sobre las rela- 
se predica del derecho comunitario adoptado por la Unión «en el ejercicio de sus competencias», mientras que en la citada Memoria de 2003 el Consejo sí se refería al mismo cuando señalaba que «no cabe oponer a lo dicho la incompetencia de las instituciones de la Unión en determinadas materias, dada la indeterminación de la distribución de competencias en los Tratados hoy vigentes y en el propio proyecto constitucional».

Así entendido el principio de primacía, el Consejo considera que «podría modularse el alcance del art. I-6» interpretando, como señala la Declaración sobre el art. I-6, que la incorporación de este principio al texto del Tratado no supone ninguna alteración respecto de la situación anterior, y teniendo en cuenta también la declaración del art. I-5, de acuerdo con la cual, «la Unión respetará la identidad nacional de los Estados miembros inherente a sus estructuras políticas y constitucionales». Sin embargo, afirma el Consejo de Estado que «en todo caso, lo cierto es que su incorporación al Tratado tiene que producir unos efectos de mayor calado que la decantación de un principio por vía jurisprudencial — susceptible así de ser aplicado con flexibilidad a través del «diálogo entre jueces»— si no se quiere desvirtuar la propia eficacia del Tratado». Lo que preocupa al Consejo es, precisamente, que se haya «positivizado» en el Tratado lo que hasta ahora era un principio de construcción jurisprudencial. Por eso, concluye que «dado que no cabe la formulación de reservas o cláusulas de excepción, no puede soslayarse un potencial conflicto entre la primacía del Derecho de la Unión y la de la Constitución».

Establecida en estos términos la contradicción entre la supremacía constitucional y el principio de primacía del derecho comunitario, añade el Consejo que, además, el alcance del principio de primacía afirmado por el Tribunal de Justicia de las Comunidades no coincide exactamente con el reconocido por los Tribunales Constitucionales de algunos Estados miembros, que expresamente han establecido ciertos límites constitucionales a la eficacia de las normas de derecho comunitario en el ordenamiento interno.

En primer lugar, se refiere el Consejo al Tribunal Constitucional alemán, concretamente a las conocidas Sentencias Solange I y II, y a la Sentencia sobre el Tratado de la Unión Europea, en las que se afirma la prevalencia de los derechos fundamentales contenidos en la Ley Fundamental alemana en tanto la Comunidad Europea no contara con un catálogo de derechos, y la competencia del Tribunal alemán para controlar el respeto a los derechos en la aplicación del derecho comunitario, así como su relación de colaboración con el

ciones internacionales, según el artículo 149.1. $3^{\mathrm{a}}$ ).

16 Considera el Consejo que dicho artículo 130 tiene, en virtud de la Política Agrícola Común y de la Política Pesquera Común, un significado diferente al que probablemente le atribuyeron los constituyentes y le dieron los primeros intérpretes. El propio Tribunal Constitucional ha reconocido que la interpretación del mismo depende mucho más del artículo 2 del Tratado CEE que de los artículos 40, 131 y 138 de la Constitución.

17 Recuerda el Consejo, por ejemplo, que los llamados «derechos especiales exclusivos», que permitían a los Estados el monopolio de los servicios de interés económico general, se consideran ahora contrarios al mercado único y a la libre circulación de mano de obra, capitales y toda clase de bienes y servicios.

18 Refiriéndose a la Memoria de 1997, en la que el Consejo de Estado ya sugirió vías para estudiar una solución semejante. 
pio de subsidiaridad que se recoge en el proyecto de Tratado puede entrar en conflicto con dicha distribución de competencias. «En términos jurídicos ello parece discrepar de las previsiones del artículo 149 de la Constitución sobre distribución de competencias entre el Estado y las Comunidades Autónomas, al igual que de otros elementos del bloque de constitucionalidad, ... Ni los Estatutos de Autonomía ni la Ley reguladora de las Bases del Régimen Local, que el Tribunal Constitucional ha considerado parámetros de constitucionalidad, responden prima facie al principio de subsidiaridad». Y señala el Consejo además que «no faltan reivindicaciones doctrinales y aún políticas en tal sentido y ya el Consejo de Estado llamó la atención sobre tal extremo en su Memoria de 1993».

Pues bien, el dictamen sobre el «Tratado por el que se establece una Constitución para Europa» se centra únicamente en una de esas tres cuestiones a las que se refirió el Consejo de Estado en su Memoria de 2003. Una vez manifestada la necesidad de verificar la compatibilidad del Tratado con la Constitución, en el dictamen se afirma que el fundamental extremo en que el Tratado puede entrar en conflicto con nuestra Norma Suprema es el relativo a la supremacía de la Constitución, y a él se dedica lo que resta de esta parte del dictamen.

Comienza el Consejo recordando que «la supremacía de la Constitución es proclamada por su art. 9.1 respecto de todo el ordenamiento jurídico y por su art. 95.1 respecto del derecho internacional (como lo ha reconocido la citada Declaración del Tribunal Constitucional), fundamenta la razón de ser del Título IX de la Constitución (del Tribunal Constitucional), es garantizada por su Título X y se reconoce expresamente por el art. 27 de la Ley Orgánica del Tribunal Constitucional y por el art. 5 de la Ley Orgánica del Poder Judicial».

La contradicción con la supremacía constitucional derivaría, principalmente, del art. I-6 del Tratado, en el que ahora se recoge el principio de primacía del derecho comunitario, elaborado por el Tribunal de Justicia de las Comunidades Europeas. ${ }^{19}$ De acuerdo con este artículo, «la Constitución (europea) y el derecho adoptado por las instituciones de la Unión en el ejercicio de las competencias que se le atribuyen a ésta primarán sobre el derecho de los Estados miembros». La Declaración sobre dicho artículo, anexa al Tratado, establece que «la Conferencia hace constar que el art. I-6 refleja la jurisprudencia existente del Tribunal de Justicia de las Comunidades Europeas y del Tribunal de Primera Instancia». A la luz de estas disposiciones y de la jurisprudencia de los citados tribunales, explica el Consejo el principio de primacía diciendo que «significa que cualquier norma del derecho comunitario, no sólo del primario sino del derivado, prevalece sobre las de derecho interno cualquiera que sea el rango de éstas, incluido el constitucional. Opera, pues, contra cualquier fuente ya sea anterior o posterior al derecho comunitario y respecto tanto de los órganos jurisdiccionales como del resto de los órganos del Estado». No se hace referencia en el dictamen al alcance de dicho principio, que, como establece expresamente el art. I-6,

19 El Consejo recoge la Jurisprudencia del Tribunal de Justicia iniciada en la sentencia de 15 de julio de 1964 (Costa c. ENEL) y desarrollada en las Sentencias de 14 de diciembre de 1971 (Politi), de 13 de julio de 1972 (Comisión c. Italia), y de 9 de marzo de 1978 (Simenthal). 
exterior y las relaciones internacionales del Estado, implicaría una eventual declaración de inconstitucionalidad». Entiende el Consejo de Estado que la relevancia histórica del mismo, y los potenciales riesgos y contraindicaciones de un eventual control posterior aconsejan utilizar la vía preventiva del art. 95.2. «Si en toda ocasión procede evitar una eventual colisión entre la Constitución y el derecho internacional o comunitario, sin duda en el presente caso se hace aún más evidente la necesidad de que España inicie su andadura bajo el nuevo Tratado con la plena certidumbre, que sólo el Tribunal Constitucional puede ofrecer, de hacerlo en armonía con su propia Constitución y con estabilidad jurídica plena».

Poniéndose en el caso de que se realizara consulta al Tribunal Constitucional, recuerda el Consejo de Estado que si «se declarara la existencia de antinomia o contradicción irreductible por vía de interpretación entre alguna o algunas estipulaciones del Tratado y la Constitución, sería preciso remover el obstáculo en cuestión por el procedimiento de reforma constitucional que corresponda, ... Ello sin perjuicio, claro está, de la aplicación del art. 93, en cuanto requiere ley orgánica para autorizar la ratificación del Tratado».

Ya en este punto, y admitiendo, como posibilidad, la reforma constitucional, el Consejo de Estado hace las dos propuestas siguientes:

a) En primer lugar, apunta que «una fórmula para salvar en este caso y pro futuro eventuales problemas de compatibilidad entre la Constitución y el derecho comunitario, quizás mejor que proceder a reformas materiales puntuales cada vez que se detecte una colisión, sería, siguiendo la pauta de otros modelos constitucionales europeos, introducir en la propia Constitución (a.e con una reformulación del artículo 93) una cláusula de integración que incorpore un mecanismo que por sí solo y en sí mismo permita — con los límites de intangibilidad que se estimen irrenunciables, con los objetivos o con los requisitos formales agravados que se consideren necesarios- una apertura general del ordenamiento español al derecho comunitario y, en su virtud, se reconozca apriorísticamente la constitucionalidad — la compatibilidad con la Constitución - de dicho ordenamiento».

b) En segundo lugar, propone, para el caso en que fuera necesaria una reforma constitucional previa a la ratificación del Tratado, «—como también se ha hecho en otros Estados miembros de la Unión Europea - aprovechar la ocasión para dar cauce a otra cuestión, si no necesaria, conveniente, cual sería la de europeizar en alguna medida la Constitución española. En su texto vigente no existe ninguna mención expresa a la Unión Europea, producto sin duda de las circunstancias del periodo constituyente. No hay referencia alguna al fenómeno de la integración europea ni en el plano teleológico (como objetivo de la nación española), ni en el plano estructural (España como Estado miembro) ni en el ámbito normativoordinamental (sin perjuicio de la referencia implícita del art. 93), ni en cuanto a las implicaciones competenciales que la pertenencia a la Unión supone para la organización política (tanto respecto de poderes del Estado y órganos constitucionales como en cuanto a la estructura territorial)».

\section{EL DICTAMEN}


Tribunal de Justicia en la tarea de garantizar los derechos fundamentales frente a la Unión Europea.

En segundo lugar, recuerda el Consejo que, «en Italia, el Tribunal Constitucional ha establecido como límites de la primacía del derecho comunitario los principios generales del ordenamiento constitucional y los derechos inalienables de la persona [Sentencias 170/1984 (Granital) y 232/1989 (Fragd)]».

Y, en tercer lugar, recoge el Consejo la jurisprudencia del Tribunal Constitucional español en ese mismo sentido, haciendo referencia expresa a las sentencias en las que éste ha afirmado la vinculación de España al derecho comunitario y el reconocimiento de la primacía de este último (STC 28/1991; también SSTC 64/1991, 130/1995 y 58/2004); a la interpretación de acuerdo con la cual la infracción de una norma comunitaria por una norma interna no entraña una conculcación del art. $93 \mathrm{CE}$, es un simple conflicto de normas «infraconstitucionales», o «no constitucionales», que corresponde resolver, no al Tribunal Constitucional, sino al órgano jurisdiccional correspondiente y, en su caso, al Tribunal de Justicia (SSTC 28/1991 y 180/1993); así como al reconocimiento por parte del Tribunal Constitucional de su competencia para pronunciarse sobre actos de los poderes públicos nacionales que sean aplicación de derecho comunitario y resulten lesivos de un derecho fundamental, es decir, contrarios a la Constitución (SSTC 64/1991 y 58/2004). A continuación, el Consejo de Estado recuerda que «la declaración del Tribunal Constitucional de 1 de julio de 1992 aborda directamente la oposición Constitución-Tratado de Maastricht, afirmando la supremacía de la Constitución e impidiendo contrariar sus determinaciones».

Termina el Consejo de Estado recordando que en su Memoria de 1992 ya subrayó «la supremacía de la Constitución respecto de cualquier otra norma jurídica, sin que se pueda celebrar un tratado internacional que contenga estipulaciones contrarias a la Constitución salvo previa revisión constitucional como exige el art. 95 de la Constitución», para, finalmente, concluir que «en atención, pues, a la posible discordancia entre lo establecido en el art. I-6 del Tratado y la supremacía de la Constitución ha de ponderarse la procedencia de acudir a lo dispuesto en el art. 95.2 de la Constitución».

\section{CONSULTA AL TRIBUNAL CONSTITUCIONAL Y PROPUESTAS DE REFORMA}

De acuerdo con lo expuesto anteriormente, el Consejo de Estado estima oportuno y conveniente que se consulte al Tribunal Constitucional, a fin de que éste declare si existe o no contradicción entre las estipulaciones del Tratado y nuestra Norma Suprema, y, de esta forma, se preserve la Constitución y se garanticen, al mismo tiempo, la seguridad y estabilidad de los compromisos en el orden internacional.

Advertida la posible incompatibilidad, y «sin perjuicio de que el respeto de lo dispuesto en el Constitución podría en todo caso salvaguardarse — caso de existencia de contradicción con ella del Tratado- incluso a posteriori, dada la posibilidad de cuestionar la constitucionalidad de los tratados, una vez formen parte del ordenamiento interno (art. 96.1 CE) mediante su impugnación ante el Tribunal Constitucional (arts. 27.2c., 31 y 32 LOTC), o a través de la propia impugnación de la ley orgánica de autorización ex art. 93 CE si se hubieran excedido los límites inherentes a ésta, es evidente la perturbación que, para la política 
Finalmente, concluye el Consejo de Estado dictaminando: «1). Que la prestación del consentimiento del Estado para obligarse en virtud del Tratado por el que establece una Constitución para Europa requiere autorización de las Cortes Generales mediante ley orgánica aprobada al amparo del art. 93 de la Constitución. 2). Que, con carácter previo a la ratificación, es conveniente que se haga uso de la facultad prevista en el art. 95.2 de la Constitución para que el Tribunal Constitucional declare si existe o no contradicción entre el Tratado y la Constitución española». ${ }^{20}$

20 En la reunión del Consejo de Ministros celebrada el 5 de noviembre de 2004 se acordó requerir al Tribunal Constitucional para que se pronunciase sobre la existencia o inexistencia de contradicción entre la Constitución española y los arts. I-6, II-111 y II-112 del «Tratado por el que se establece una Constitución para Europa», así como sobre la suficiencia del art. $93 \mathrm{CE}$ para dar cauce a la prestación del consentimiento del Estado al Tratado y, en su caso, sobre el procedimiento de reforma constitucional que hubiera de seguirse para adecuar el texto de la Constitución española a dicho Tratado. El Tribunal Constitucional se pronunció mediante la Declaración 1/2004, de 13 de diciembre. 
